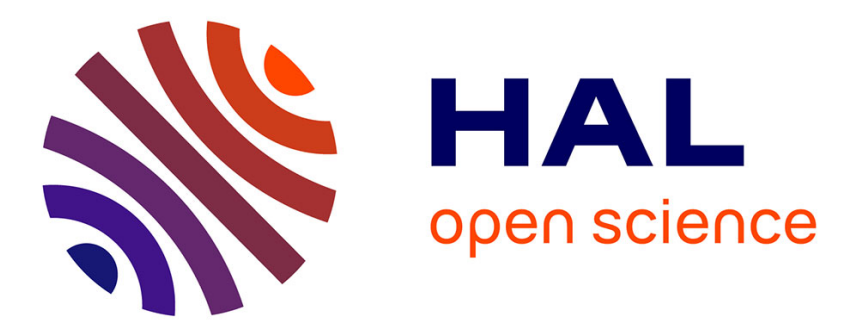

\title{
Mécanismes de décomposition catalytique dans un plasma d'ammoniac basse pression. Etude cinétique en présence d'argon comme actinomètre
}

\author{
A. Gicquel, P. Saillard, N. Laidani, J. Amouroux
}

\section{- To cite this version:}

A. Gicquel, P. Saillard, N. Laidani, J. Amouroux. Mécanismes de décomposition catalytique dans un plasma d'ammoniac basse pression. Etude cinétique en présence d'argon comme actinomètre. Revue de Physique Appliquée, 1989, 24 (3), pp.285-294. 10.1051/rphysap:01989002403028500 . jpa-00246050

\section{HAL Id: jpa-00246050 https://hal.science/jpa-00246050}

Submitted on 1 Jan 1989

HAL is a multi-disciplinary open access archive for the deposit and dissemination of scientific research documents, whether they are published or not. The documents may come from teaching and research institutions in France or abroad, or from public or private research centers.
L'archive ouverte pluridisciplinaire HAL, est destinée au dépôt et à la diffusion de documents scientifiques de niveau recherche, publiés ou non, émanant des établissements d'enseignement et de recherche français ou étrangers, des laboratoires publics ou privés. 


\title{
Mécanismes de décomposition catalytique dans un plasma d'ammoniac basse pression. Etude cinétique en présence d'argon comme actinomètre
}

\author{
A. Gicquel, P. Saillard, N. Laidani et J. Amouroux \\ Laboratoire de Génie Chimique, Equipe des Réacteurs Chimiques en phase Plasma, E.N.S.C.P., 11 rue Pierre \\ et Marie Curie, 75231 Paris Cedex 05, France
}

(Reçu le 8 décembre 1987, révisé le 5 septembre 1988, accepté le 22 novembre 1988)

\begin{abstract}
Résumé. - La décomposition de l'ammoniac a été étudiée dans un réacteur tubulaire sous plasma basse pression. Les paramètres clés de la transformation sont, outre la densité électronique et l'énergie cinétique des électrons, l'excitation vibrationnelle du plasma et le temps de séjour des espèces. L'effet catalytique du titane sur la décomposition de $\mathrm{NH}_{3}$ a pu être corrélé à la formation d'une couche limite à l'interface plasma-substrat, caractérisée par une forte excitation vibrationnelle. Ceci met en évidence l'existence de transferts énergétiques particuliers entre la phase gazeuse et le substrat, responsables de l'augmentation de la réactivité chimique dans cette zone.
\end{abstract}

\begin{abstract}
NH}_{3}$ decomposition has been studied in a tubular reactor under low pressure plasma conditions. The key parameters for this transformation are, besides the electron density and kinetic energy, the vibrational excitation and the residence time. The catalytic effect of titanium on the $\mathrm{NH}_{3}$ decomposition has been correlated to the formation of a boundary layer characterized by a strong vibrational excitation. This gives evidence for particular energetic transfers between the gaseous phase and the substrate, responsible for an increase of the chemical reactivity in that zone.
\end{abstract}

\section{Introduction.}

Dans un milieu hors équilibre, la répartition énergétique entre les différents modes d'excitation de la molécule influe sur sa réactivité chimique. C'est ainsi que Polanyi (Réf. [1]) a montré qu'en phase gazeuse homogène, une forte excitation vibrationnelle des réactifs favorise les processus élémentaires de type endothermique ; les produits de la réaction sont alors rotationnellement excités. Des travaux antérieurs effectués au laboratoire (Réfs. $[2,5,16])$ ont permis d'établir que les lois de Polanyi sont applicables aux milieux complexes tels que les plasmas hors équilibre. Nous avons pu ainsi montrer que la décomposition de $\mathrm{NH}_{3}$ en phase gazeuse est contrôlée par une étape endothermique, de type $\mathrm{NH}_{3}(v)+\mathrm{e}^{-} \rightarrow \mathrm{NH}_{x}(J)+\mathrm{H}_{3-x}+\mathrm{e}^{-}$, puisqu'une diminution de pression de 20 à 5 mbars accentue le caractère hors équilibre du plasma ( $T_{\text {vib }}$ augmente de 3000 à $3300 \mathrm{~K}$ et $T_{\text {rot }}$ diminue de 1207 à $817 \mathrm{~K}$ ); simultanément, le taux de décomposition de $\mathrm{NH}_{3}$ augmente de 34 à $42 \%$. La décomposition de
$\mathrm{NH}_{3}$ est donc contrôlée, en milieu plasmagène, par l'excitation vibrationnelle et non par l'excitation rotationnelle.

L'effet catalytique du titane est mis en évidence par une augmentation du taux de décomposition de $\mathrm{NH}_{3}$. Simultanément, on observe la formation d'une couche limite gazeuse à l'interface plasma/surface, dont les caractéristiques diffèrent de celles de la veine gazeuse principale et témoignent de phénomènes d'échanges entre la phase gazeuse et le substrat (Réf. [5]). Par ailleurs, la formation d'une couche de nitrure sur la surface de titane, dont la cinétique de croissance dépend des conditions expérimentales, a été mise en évidence (Réf. [15]). Le nitrure métallique constitue, en réalité, plus probablement le catalyseur plutôt que le métal proprement dit.

Une analyse systématique de la couche limite permet d'établir une corrélation entre le pouvoir catalytique du substrat et les propriétés physicochimiques de la couche limite, et ainsi d'accéder aux mécanismes réactionnels responsables de la catalyse hétérogène dans de tels milieux. 
2. Dispositif expérimental, définitions des paramètres et diagnostics d'analyse.

Le dispositif expérimental est représenté sur la figure 1 . Le plasma est créé dans un réacteur tubulaire en quartz (silice fondue) (40 cm de longueur) par une décharge radiofréquence inductive oscillant à $40 \mathrm{MHz}$, dont la puissance varie de 200 à $1000 \mathrm{~W}$. L'échantillon de titane est positionné perpendiculairement au flux gazeux et il est relié à une trempe (paroi refroidie à l'eau) par l'intermédiaire d'une tige en titane de distance variable. Un couplage capacitif s'établit entre la borne portée à la haute tension et l'échantillon de titane, il s'ensuit que le volume de la décharge proprement dit est défini par le volume compris entre cette borne et soit la tête de trempe, soit le substrat.

Deux réacteurs de diamètre respectivement de 4,5 et $10 \mathrm{~cm}$ ont été utilisés dans cette étude afin de faire varier le temps de séjour des espèces dans la phase gazeuse et la densité d'énergie du plasma dans des gammes assez larges.

Typiquement, pour le réacteur de $4,5 \mathrm{~cm}$ de diamètre, le volume plasma est de 286 et de $243 \mathrm{~cm}^{3}$ respectivement en absence et en présence de substrat, cependant une expérience a été réalisée avec un volume de $43 \mathrm{~cm}^{3}$ (par réduction de la distance spire-substrat) dans le but d'obtenir une forte densité d'énergie $\left(65 \times 10^{-17} \mathrm{~W} /\right.$ molécule $)$ associée à un très faible temps de séjour $(4 \mathrm{~ms})$. Pour le réacteur de $10 \mathrm{~cm}$ de diamètre, le volume plasma est de $785 \mathrm{~cm}^{3}$.

Le temps de séjour (s) dans la phase gazeuse, entre les spires et le substrat, est défini par la relation (Réf. [17]) :

$$
\tau_{\mathrm{s}}=18 \times 10^{-3} \Pi\left(r^{2} d / D\right)\left(P / T_{\mathrm{g}}\right)(1 /(1+\bar{X}))
$$

dans laquelle $r$ est le rayon du réacteur (en $\mathrm{cm}) ; d$, la longueur de la décharge (en $\mathrm{cm}) ; D$, le débit (en $1 /$ min) $; P$, la pression (en mbars) $; T_{\mathrm{g}}$, la température du gaz assimilée à la température de rotation de $\mathrm{N}_{2}\left(\mathrm{C}^{3} \Pi_{\mathrm{u}}\right)$ (en $\left.\mathrm{K}\right)$ et $\bar{X}$, la valeur moyenne du taux de conversion, assimilée à $X / 2$, tenant compte de l'augmentation du nombre de moles lors de la réaction (augmentation de pression). Il convient de noter que le temps de séjour est ici égal au temps de passage dans le milieu gazeux et que le temps de séjour des espèces sur la surface n'a pas été pris en compte.

La densité d'énergie ( $P_{1} / N$ en $\mathrm{W} /$ molécule) est définie par le rapport de la puissance injectée et du nombre total de molécules $(N)$ dans le volume de la décharge. Pour le calcul de $N$, la température du gaz

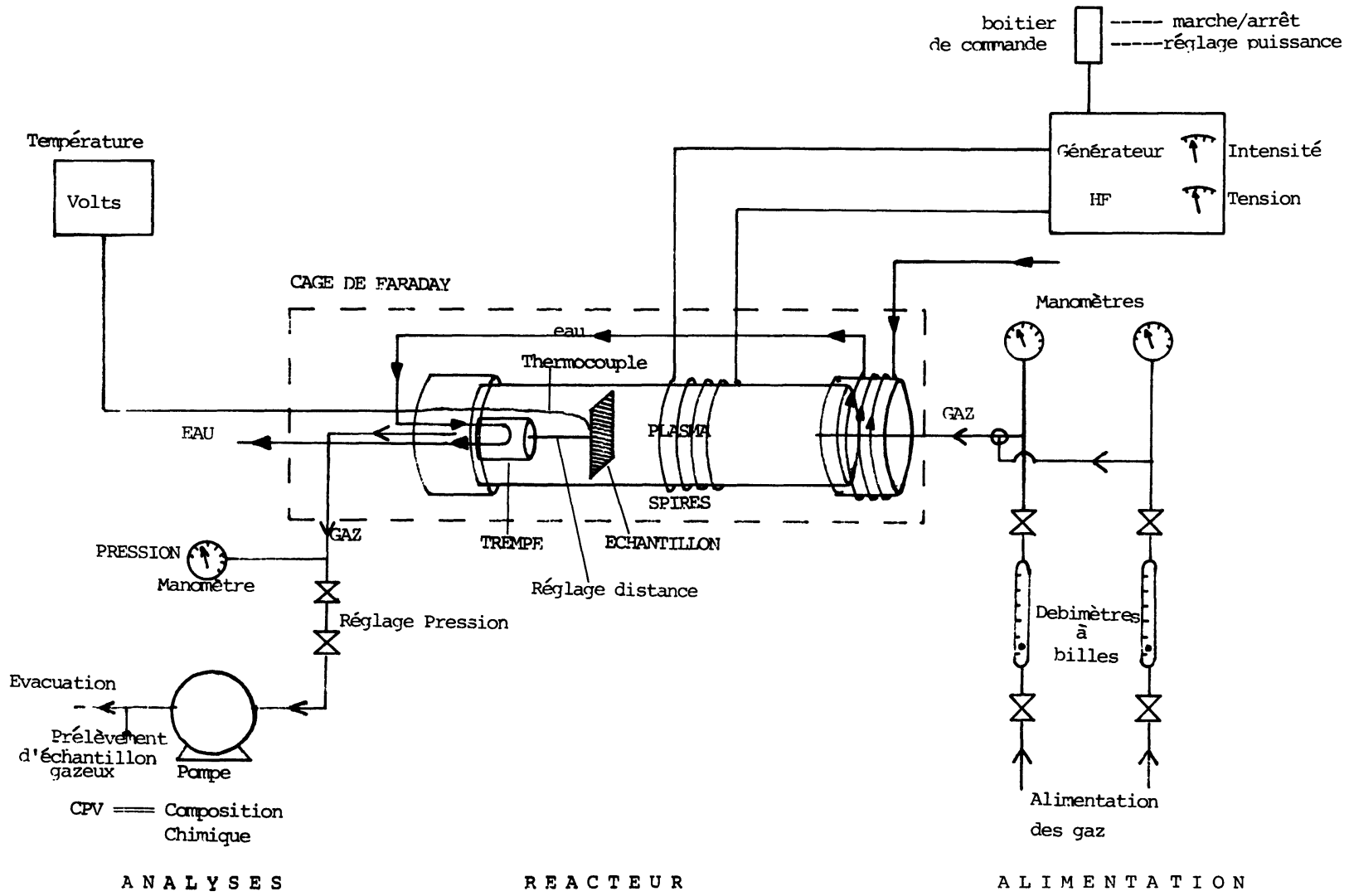

Fig. 1. - Schéma expérimental.

[Experimental apparatus.] 
a été assimilée à la température de rotation de $\mathrm{N}_{2}\left(C^{3} \Pi_{\mathrm{u}}\right)$ et le gaz est supposé parfait.

$$
P_{\mathrm{I}} / N=\text { Puissance injectée } \times R T_{\text {rot }} /(P \times V) \text {. }
$$

Les diagnostics utilisés, caractérisant la phase gazeuse sont :

- la chromatographie en phase gazeuse donnant accès au taux de décomposition de $\mathrm{NH}_{3}(\mathrm{X})$ (précision $\pm 2 \%$ ) ;

- l'analyse spectroscopique de l'émission visible et UV du plasma, tranche par tranche le long du réacteur ; les transitions plus particulièrement analysées étant :

* $\mathrm{C}^{3} \Pi_{\mathrm{u}} \rightarrow \mathrm{B}^{3} \Pi_{\mathrm{g}}(\Delta v=-2)$ de $\mathrm{N}_{2}$ donnant accès à la température de vibration de cet état (précision de la mesure $\pm 80 \mathrm{~K}$ ) ; cette température peut être considérée comme une image de celle représentant la distribution vibrationnelle de l'état fondamental dans la mesure où l'on s'est assuré que l'état $\mathrm{C}^{3} \Pi_{\mathrm{u}}$ est peuplé par impact électronique direct (Réf. [4]).

* $\mathrm{C}^{3} \Pi_{\mathrm{u}} \rightarrow \mathrm{B}^{3} \Pi_{\mathrm{g}}(\Delta v=0)$ de $\mathrm{N}_{2}$ donnant accès à la température de rotation de cet état (précision de la mesure $\pm 50 \mathrm{~K}$ ), qui devrait pouvoir être assimilée à la température de rotation de l'état fondamental et à la température cinétique du gaz par suite des transferts respectivement rotation-rotation et rotation-translation entre les molécules d'azote très rapides dans la gamme de pression étudiée ;

* $\mathrm{A}^{3} \Pi \rightarrow \mathrm{X}^{3} \Sigma^{-}$de $\mathrm{NH}$, donnant accès à la variation de l'intensité d'émission de cet état le long du réacteur ;

* actinométrie (Réfs. $[3,18])$ : nous avons vérifié, dans un premier temps, qu'une introduction d'argon dans la décharge ( $9 \%$ ) ne modifie pas les caractéristiques énergétiques du plasma (variation de l'intensité d'émission de $\mathrm{N}_{2}^{*}$ inférieure à $5 \%$ ).

Dans un deuxième temps nous nous sommes assurés que, dans ces conditions expérimentales, en particulier dans la couche limite, $\mathrm{N}_{2}\left(\mathrm{C}^{3} \Pi_{\mathrm{u}}\right)$ est peuplé directement à partir de l'état fondamental $\mathrm{N}_{2}(X)$ par collision avec un électron libre. Nous avons fait varier la concentration de $\mathrm{N}_{2}$ dans le réacteur en introduisant de l'hélium comme gaz diluant et nous obtenons une relation linéaire entre le rapport des intensités d'émission de $\mathrm{N}_{2}$ et de l'argon et le rapport de leurs concentrations respectives (Fig. 2). La concentration de $\mathrm{N}_{2}$ dans la couche limite est proportionnelle à $X \times\left[\mathrm{NH}_{3}\right]$, où $X$ rep̈résente le taux de décomposition de $\mathrm{NH}_{3}$. L'obtention de la linéarité entre le rapport des intensités d'émission et des concentrations mérite quelques commentaires. En effet, l'introduction d'hélium dans la décharge est connue pour modifier les paramètres du plasma, en particulier pour augmenter la moyenne d'énergie des électrons et / ou la densité électronique, ce qui peut avoir une influence sur le

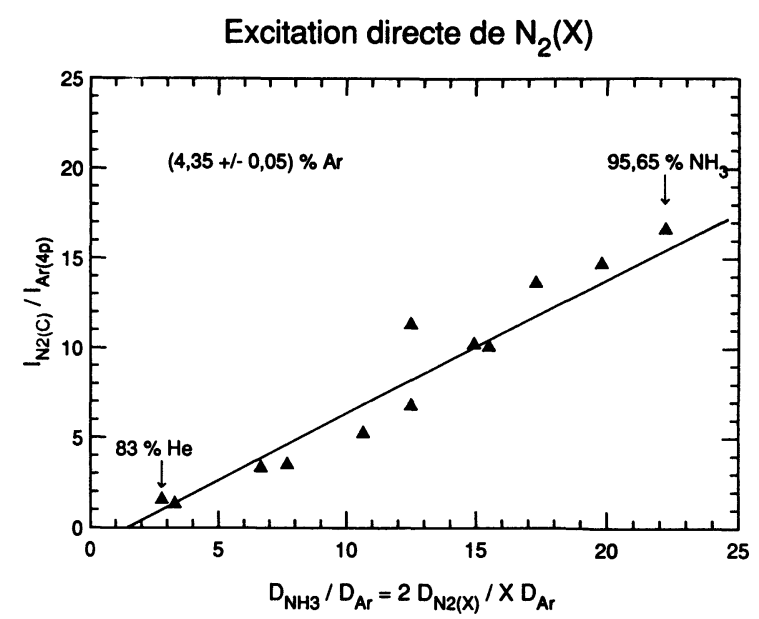

Fig. 2. - Variation du rapport des intensités d'émission de $\mathrm{N}_{2}(\mathrm{C} \rightarrow \mathrm{B})$ et de l'argon $(4 \mathrm{p} \rightarrow 4 \mathrm{~s})$ en fonction de leur rapport en flux. Le flux $\mathrm{D}_{\mathrm{N}_{2}}=X \times \mathrm{D}_{\mathrm{NH}_{3}} / 2$, où $X$ est le taux de décomposition de $\mathrm{NH}_{3}$.

[Variation of the ratio of emission intensities of $\mathrm{N}_{2}(\mathrm{C} \rightarrow \mathrm{B})$ and argon $(4 \mathrm{p} \rightarrow 4 \mathrm{~s})$ as a function of their flow rate ratio. $\mathrm{D}_{\mathrm{N}_{2}}=X \times \mathrm{D}_{\mathrm{NH}_{3}} / 2$, where $X$ is the $\mathrm{NH}_{3}$ decomposition rate.]

taux de décomposition de $\mathrm{NH}_{3}$. Cependant l'effet de la dilution est également d'une part, de limiter les réactions de recombinaison de type $\mathrm{NH}+\mathrm{NH} \rightarrow \mathrm{N}_{2}+\mathrm{H}_{2}$ et d'autre part, d'avoir deux effets antagonistes sur l'excitation vibrationnelle; le premier est d'augmenter celle-ci grâce à l'augmentation de l'énergie des électrons et/ou de leur densité, le deuxième est de diminuer celle-ci par augmentation du nombre d'atomes conduisant à des transferts d'énergie vibration-translation. L'ensemble de ces processus conduit, compte tenu des résultats obtenus, à soit une invariance du taux de décomposition de $\mathrm{NH}_{3}$ lors de l'introduction de l'hélium, soit à une évolution linéaire de celui-ci en fonction de la concentration d'hélium introduit.

Il était important de vérifier que $\mathrm{N}_{2}\left(\mathrm{C}^{3} \Pi_{\mathrm{u}}\right)$ est bien peuplé par impact électronique direct dans la couche limite dans la mesure où il est connu que la recombinaison d'atomes d'azote sur des parois métalliques produit efficacement des molécules d'azote dans l'état $\mathrm{B}{ }^{3} \Pi_{\mathrm{g}}$ à des niveaux vibrationnels excités (Réfs. [21-23]), cependant dans ces conditions, il semblerait que ce n'est pas le cas. L'interprétation de ce phénomène n'est pas clair, cependant il est probable que la réaction de surface principale provienne de la recombinaison des radicaux $\mathrm{NH}$ par des processus de chimisorption-désorption. Par ailleurs, dans le cas où la réaction principale proviendrait de la recombinaison des atomes d'azote, les nouvelles molécules désorberaient dans un milieu plus complexe chimiquement qu'un milieu ne contenant que des molécules et des atomes d'azote (milieux dans lesquels la formation de l'état $\mathrm{B}{ }^{3} \Pi_{\mathrm{g}}$ est généralement observée). 
Une fois ces deux vérifications effectuées, nous avons mesuré l'évolution du rapport des intensités d'émission des transitions $\mathrm{C}^{3} \Pi_{\mathrm{u}} \rightarrow \mathrm{B}^{3} \Pi_{\mathrm{g}}$ de $\mathrm{N}_{2}$ et $4 \mathrm{p} \rightarrow 4 \mathrm{~s}$ de l'argon. Ce rapport donne alors accès à l'évolution de la densité relative de l'azote moléculaire à l'état fondamental le long du réacteur et en fonction des conditions expérimentales (Réf. [5]). Rappelons que pour cela, le temps de relaxation radiative doit être très inférieur au temps de collision molécule-molécule (induisant des transferts d'énergie entre particules) et au temps de collision électron-molécule (pouvant dépeupler l'état observé en l'excitant sur un niveau supérieur).

\section{Analyse de la phase gazeuse. Détermination des paramètres clés de la décomposition de $\mathbf{N H}_{\mathbf{3}}$.}

Les variations de la température de vibration de $\mathrm{N}_{2}(\mathrm{C})$ avec la densité énergétique du plasma $P_{\mathrm{I}} / N$ (watt/molécule), et le temps de séjour des espèces, $\tau_{\mathrm{s}}(\mathrm{ms})$, sont illustrées par la figure 3 . Une augmentation de la densité énergétique du plasma de 5,5 à $39 \times 10^{-17} \mathrm{~W} /$ molécule augmente la température de vibration de 3200 à $3700 \mathrm{~K}$, pour un temps de séjour d'environ 20 à $30 \mathrm{~ms}$. Par ailleurs, une augmentation du temps de séjour de 26 à $55 \mathrm{~ms}$ conduit à une augmentation de la température de vibration de 3300 à $3450 \mathrm{~K}$, pour une densité énergétique de $9 \times 10^{-17} \mathrm{~W} /$ molécule. Par conséquent, la densité énergétique du plasma et le temps de séjour sont les paramètres clés pour l'excitation vibrationnelle de $\mathrm{N}_{2}(C)$.

L'influence de ces mêmes paramètres sur la réactivité chimique du milieu est illustrée par la figure 4 . Le taux de décomposition de $\mathrm{NH}_{3}$ augmente avec la densité d'énergie du plasma et avec le temps de séjour. Ces résultats permettent de relier la variation

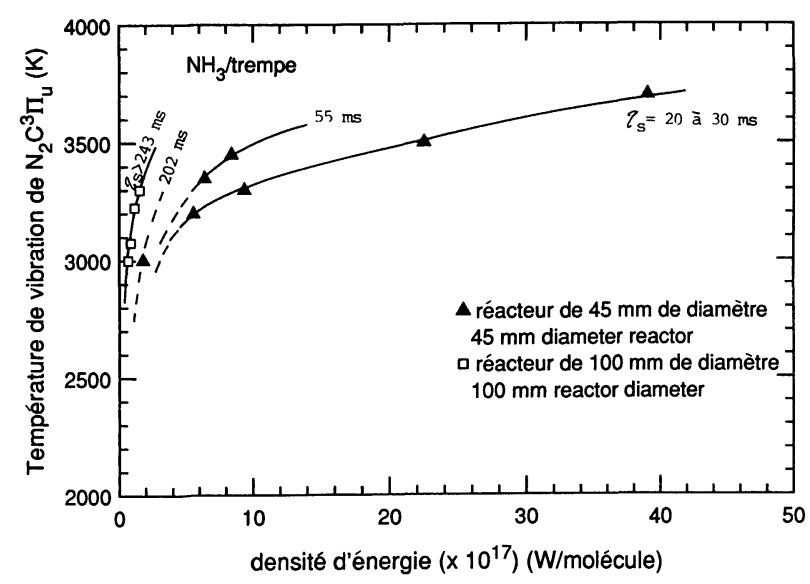

Fig. 3. - Température de vibration de $\mathrm{N}_{2}\left(\mathrm{C}^{3} \Pi_{\mathrm{u}}\right)$ en fonction de la densité énergétique du plasma, à temps de séjour constant.

[Vibrational temperature of $\mathrm{N}_{2}\left(\mathrm{C}^{3} \Pi_{\mathrm{u}}\right)$ versus plasma energetic density, for constant residence time.]

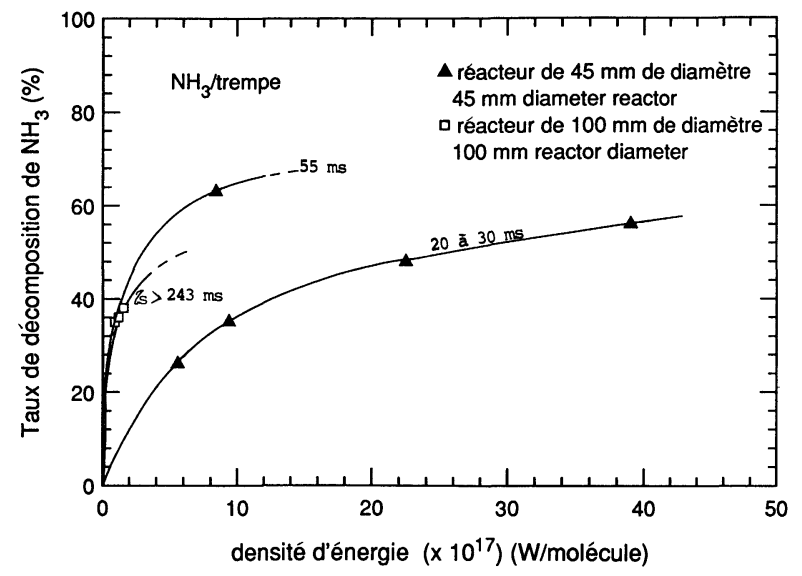

Fig. 4. - Evolution du taux de décomposition de $\mathrm{NH}_{3}$ en fonction de la densité énergétique du plasma, $P_{\mathrm{I}} / N$, à temps de séjour constant.

$\left[\mathrm{NH}_{3}\right.$ decomposition rate versus plasma energetic density, for constant residence time.]

du taux de décomposition de $\mathrm{NH}_{3}$ d'une part, à la température de vibration de $\mathrm{N}_{2}(\mathrm{C})$ (Fig. 5), et d'autre part, au temps de séjour pour différentes températures de vibration (Fig. 6).

Ces courbes expérimentales présentent une grande similitude avec les courbes théoriques isotemps, $X=f(T)$, et isotherme, $X=f(t)$ correspondant à un système chimique contrôlé par une étape endothermique dans un réacteur en phase homogène à l'équilibre thermodynamique (Fig. 7, Réf. [7]). Il est alors possible de conclure que la décomposition de $\mathrm{NH}_{3}$ dans un réacteur hors équilibre suit les variations correspondant au cas de réacteurs tubulaires contrôlés par une étape endothermique à condition de ne plus considérer la température du gaz,

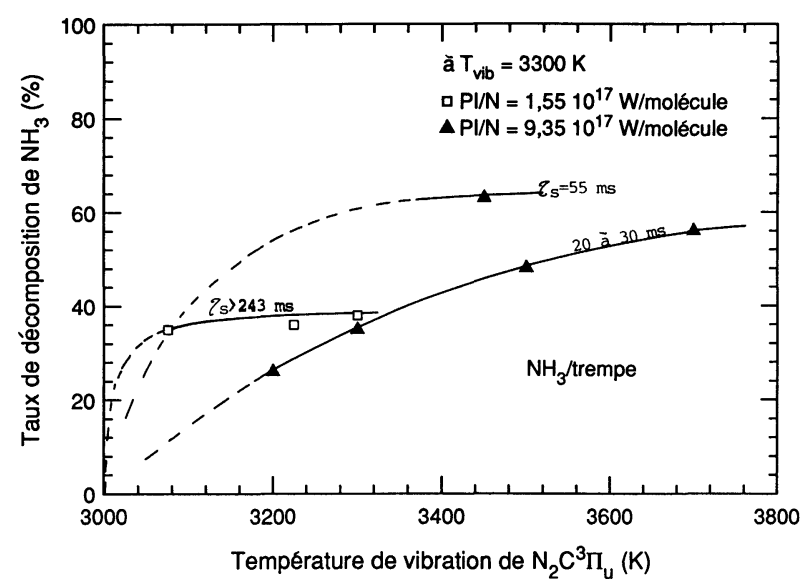

Fig. 5. - Evolution du taux de décomposition de $\mathrm{NH}_{3}$ en fonction de la température de vibration de $\mathrm{N}_{2}\left(\mathrm{C}^{3} \Pi_{\mathrm{u}}\right)$, à temps de séjour constant.

$\left[\mathrm{NH}_{3}\right.$ decomposition rate versus vibrational temperature of $\mathrm{N}_{2}\left(\mathrm{C}^{3} \Pi_{\mathrm{u}}\right)$, for constant residence time.] 


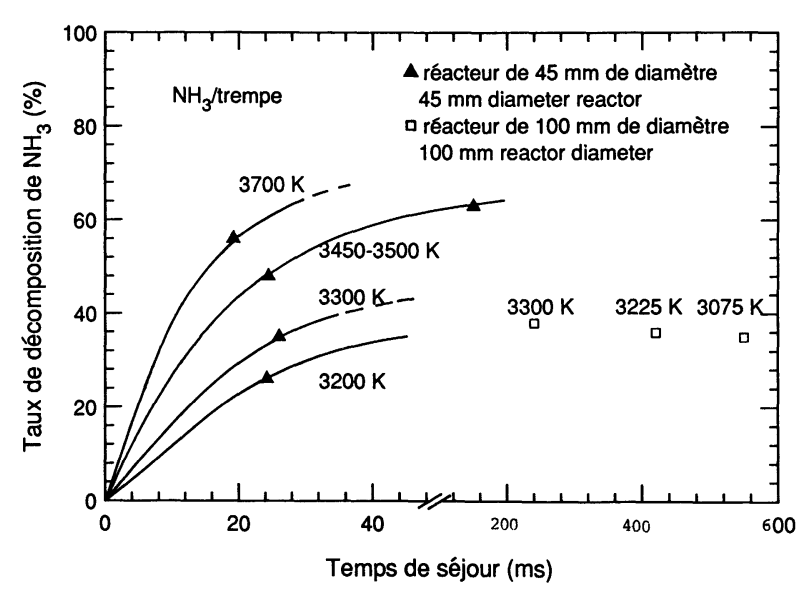

Fig. 6. - Evolution du taux de décomposition de $\mathrm{NH}_{3}$ en fonction du temps de séjour, à température de vibration de $\mathrm{N}_{2}\left(C^{3} \Pi_{\mathrm{u}}\right)$ constante.

$\left[\mathrm{NH}_{3}\right.$ decomposition rate versus residence time, for constant vibrational temperature of $\mathrm{N}_{2}\left(\mathrm{C}^{3} \Pi_{\mathrm{u}}\right)$.]

mais une température de vibration rendant compte de l'excitation vibrationnelle des espèces réactives comme paramètre clé.

Bien que dans notre cas, nous n'ayons pas accès directement à la température de vibration de $\mathrm{NH}_{3}$, la variation du taux de décomposition de $\mathrm{NH}_{3}$ en fonction de la température de vibration de $\mathrm{N}_{2}(\mathrm{C})$ traduit le fait que les transferts d'énergie vibration-vibration de $\mathrm{N}_{2}(X, v)$ vers $\mathrm{NH}_{3}$ sont efficaces, ce qui est en accord avec Back et Salahub (Réf. [6]).

Le temps de séjour constitue un second paramètre déterminant dans ce type de décharge. En effet, le taux de décomposition de $\mathrm{NH}_{3}$ augmente de 48 à $55 \%$ lorsque le temps de séjour augmente de 25 à
$55 \mathrm{~ms}$, la température de vibration étant maintenue comprise entre 3450 et $3500 \mathrm{~K}$ (Fig. 6).

L'étude entreprise ayant permis de déterminer les paramètres clés de la décomposition, il apparaît désormais possible d'envisager dans le futur une modélisation des réacteurs à décharge basse pression contrôlés par une étape endothermique.

Par ailleurs, la comparaison des résultats, d'une part à faible densité énergétique $\left(P_{\mathrm{I}} / N=1,55 \times\right.$ $10^{-17} \mathrm{~W} /$ molécule) et grand temps de séjour $\left(\tau_{\mathrm{s}}=243 \mathrm{~ms}\right)$, et d'autre part à plus forte densité d'énergie $\left(P_{\mathrm{I}} / N=9,35 \times 10^{-17} \mathrm{~W} /\right.$ molécule $)$ et faible temps de séjour $\left(\tau_{\mathrm{s}}=26 \mathrm{~ms}\right)$, met en évidence le rôle de l'énergie et/ou de la densité des électrons vis-à-vis de la dissociation primaire de $\mathrm{NH}_{3}$. Pour une température de vibration constante $(3300 \mathrm{~K})$, le taux de conversion est pratiquement le même bien que la densité énergétique varie d'un facteur 6 (Fig. 5). Cependant, la densité électronique $\left(n_{\mathrm{e}}\right)$ et la température électronique $\left(T_{\mathrm{e}}\right)$ de nos plasmas n'ont pas été mesurées expérimentalement, ce qui ne permet pas d'établir une relation précise. On peut seulement admettre que $n_{\mathrm{e}}$ et $T_{\mathrm{e}}$ sont respectivement de l'ordre de $10^{9}$ à $10^{11} \mathrm{~cm}^{-3}$ (Réf. [8]) et de $13000 \mathrm{~K}$ (Réf. [9]).

\section{Effet catalytique du titane.}

L'introduction d'un échantillon de titane dans la décharge conduit à une augmentation du taux de décomposition de $\mathrm{NH}_{3}$ de $35 \%$ en absence de titane (Fig. 6) à $45 \%$ en présence de titane (Fig. 8), pour les mêmes conditions expérimentales $\left(\tau_{\mathrm{s}}=21 \mathrm{~ms}\right.$, $T_{\text {vib }}=3300 \mathrm{~K}, P_{\mathrm{I}} / N=10,9 \times 10^{-17} \mathrm{~W} /$ molécule $)$. Par conséquent, le titane catalyse la réaction de décomposition de $\mathrm{NH}_{3}$.

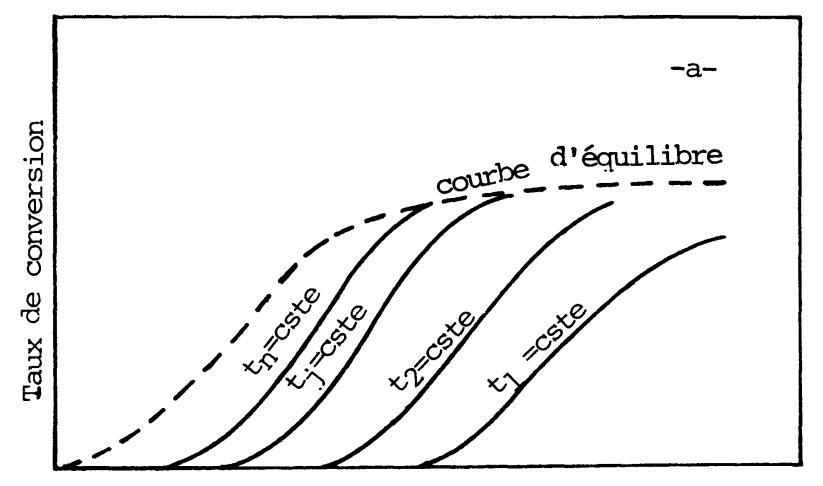

Température (K)

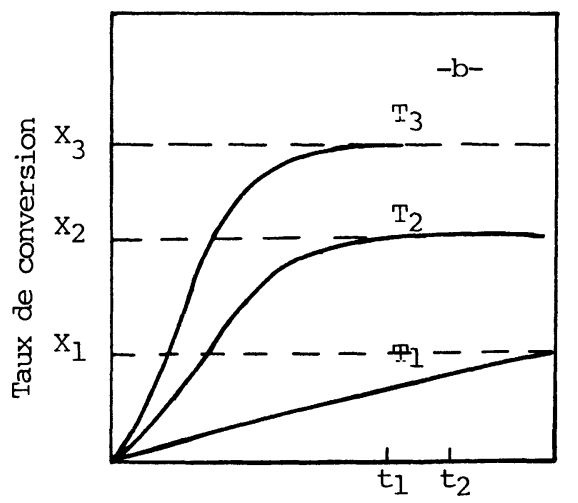

temps de sejour

Fig. 7. - a) Courbes isotemps calculées, $X=f(T)$, pour un système contrôlé par un processus endothermique dans un réacteur tubulaire (Réf. [7]). b) Courbes isothermes calculées, $X=f\left(t_{\mathrm{s}}\right)$, pour un système contrôlé par un processus endothermique.

[a) Isotime calculated curves, $X=f(T)$, for a system controlled by an endothermic step in a tubular reactor (Ref. [7]).

b) Isotherm calculated curves, $X=f\left(t_{\mathrm{s}}\right)$, for a system controlled by an endothermic step.] 


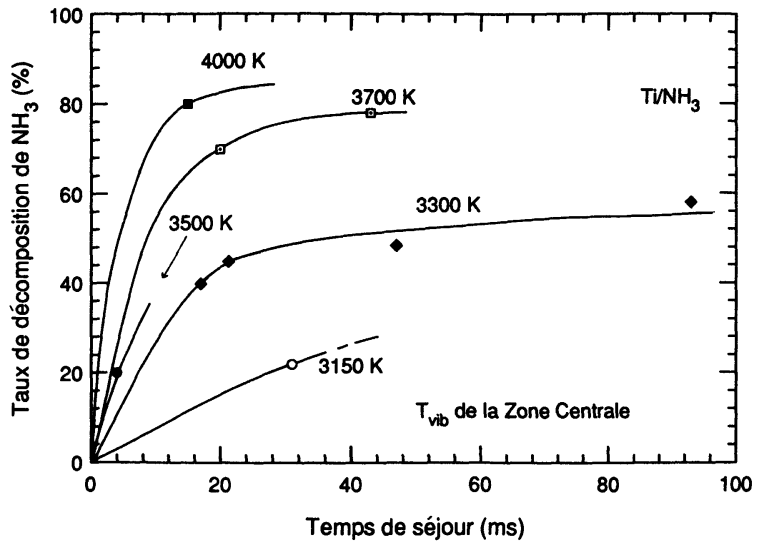

Fig. 8. - Faisceau de courbes isothermes (température de vibration de la zone centrale) du taux de décomposition de $\mathrm{NH}_{3}$ en fonction du temps de séjour, en présence d'une surface de titane.

[Isotherm curves (vibrational temperature of the plasma bulk) of the decomposition rate versus residence time, in presence of a titanium surface.]

L'analyse spectroscopique le long du réacteur révèle simultanément, la formation d'une couche limite à l'interface plasma-substrat caractérisée par une forte température de vibration de $\mathrm{N}_{2}\left(\mathrm{C}^{3} \Pi_{\mathrm{u}}\right)$ (Fig. 9). La température de vibration est, en effet, plus élevée dans la couche limite $(3800 \mathrm{~K})$ que dans le cour du plasma ( $3300 \mathrm{~K})$ et le titane n'a aucune influence sur la valeur de l'excitation vibrationnelle du coeur du plasma.

L'apparition de cette zone à l'interface plasma/surface, dont le caractère hors équilibre
$\left(T_{\text {vib }}-T_{\text {rot }}=2550 \mathrm{~K}\right)$ est plus marqué que celui de la veine gazeuse principale $\left(T_{\mathrm{vib}}-T_{\mathrm{rot}}=1700 \mathrm{~K}\right)$, met en évidence l'existence de transferts énergétiques particuliers entre la phase gazeuse et le substrat.

L'utilisation de l'argon en actinomètre établit que la couche limite constitue un réacteur de recombinaison produisant de l'azote moléculaire dans l'état électronique fondamental puisque le rapport $I_{\mathrm{N}_{2}} / I_{\mathrm{Ar}}$ est cinq fois plus élevé dans la couche limite que dans la zone centrale (Fig. 10). En outre, les résultats mettent en évidence le rôle catalytique de la surface de titane par rapport à la paroi de trempe (refroidie à l'eau), dans la mesure où le rapport $I_{\mathrm{N}_{2}} / I_{\mathrm{Ar}}$ augmente d'un rapport 1,5 entre l'interface plasma/trempe et l'interface plasma/titane (Fig. 10). Il convient de remarquer que les molécules d'azote produites par la réaction de surface sont vibrationnellement excitées ; elles sont, par ailleurs, ultérieurement rapidement excitées électroniquement dans la couche limite via des collisions molécule-électrons énergétiques, puisque la couche limite possède un caractère de chute cathodique.

Ainsi, bien que le volume du réacteur catalytique correspondant à la couche limite soit faible devant le volume de la zone centrale, sa contribution à la réaction globale de décomposition de $\mathrm{NH}_{3}$ est importante. Sa contribution dépend cependant des paramètres de la décharge (densité d'énergie, pression, temps de séjour) puisque les dimensions et les caractéristiques de la couche limite et la température du substrat sont des fonctions de ces paramètres. Par ailleurs, le temps de séjour au voisinage de la surface dépend du flux gazeux, de la viscosité et de la

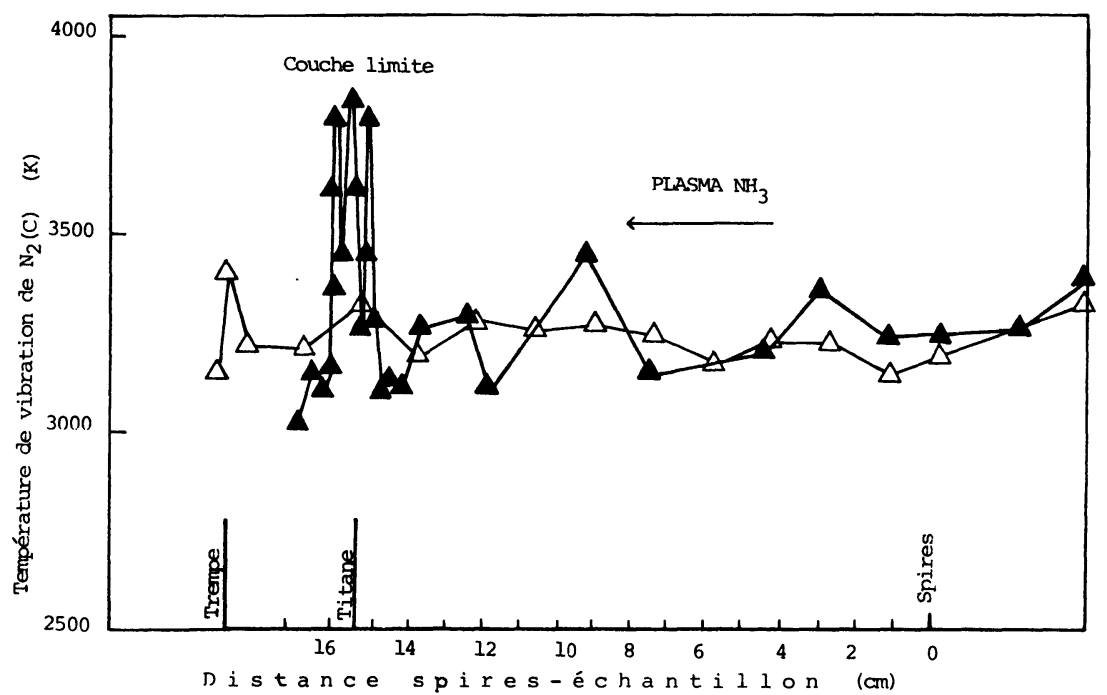

Fig. 9. - Influence de l'introduction d'une surface de titane sur l'évolution de la température de vibration de $\mathrm{N}_{2}\left(C^{3} \Pi_{u}\right)$ le long du réacteur à plasma d'ammoniac: en présence d'une surface de titane, en absence du titane.

$$
\left(P=5 \text { mbars, } P_{\mathrm{I}}=780 \mathrm{~W}, P_{\mathrm{I}} / N=10 \times 10^{-17} \mathrm{~W} / \mathrm{molécule,} D=0,67 \mathrm{l} / \mathrm{min}\right) \text {. }
$$

[Role of a titanium target on the vibrational temperature of $\mathrm{N}_{2}\left(\mathrm{C}^{3} \Pi_{\mathrm{u}}\right)$, along the $\mathrm{NH}_{3}$ plasma reactor : in presence of a titanium target, in absence of titanium.

$$
\left.\left(P=5 \mathrm{mbars}, P_{\mathrm{I}}=780 \mathrm{~W}, P_{\mathrm{I}} / N=10 \times 10^{-17} \mathrm{~W} / \mathrm{molecule}, D=0.67 \mathrm{l} / \mathrm{min}\right) .\right]
$$




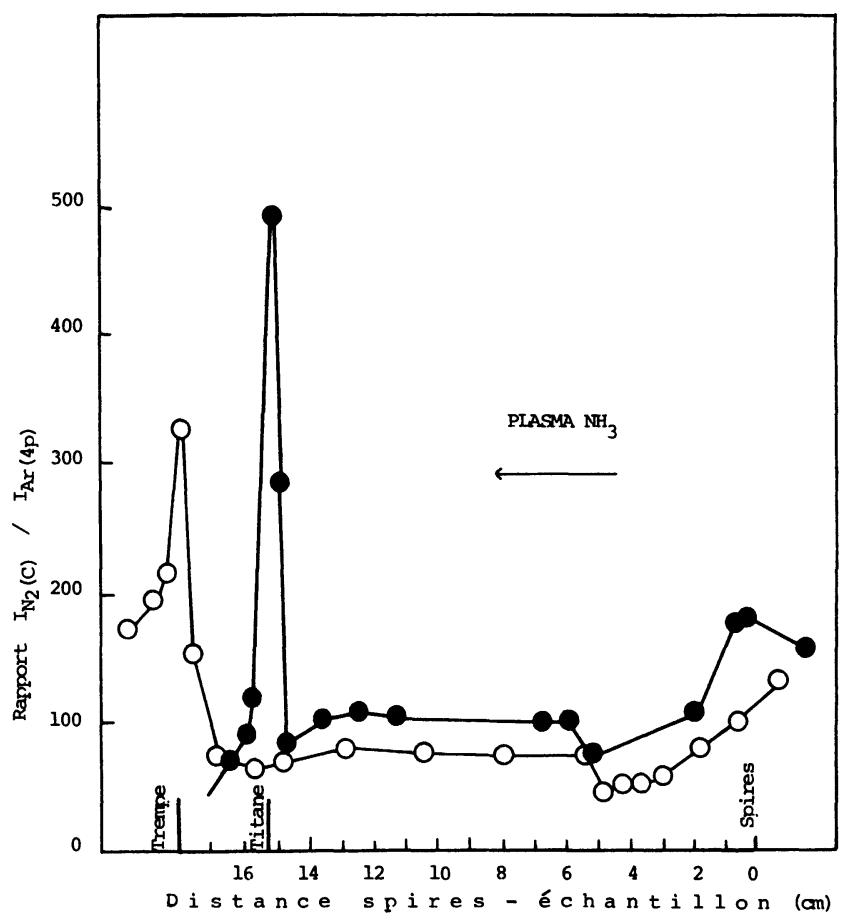

Fig. 10. - Influence de l'introduction d'une surface de titane sur l'évolution du rapport $I_{\mathrm{N}_{2}(\mathrm{C})} / I_{\mathrm{Ar}(4 \mathrm{p})}$, le long du réacteur : en présence d'une surface de titane ; en absence du titane.

( $P=5$ mbars, $P_{\mathrm{I}}=780 \mathrm{~W}$,

$$
\left.P_{\mathrm{I}} / N=10 \times 10^{-17} \mathrm{~W} / \text { molécule, } D=0,67 \mathrm{l} / \mathrm{min}\right) .
$$

[Role of a titanium target on the $I_{\mathrm{N}_{2}(\mathrm{C})} / I_{\mathrm{Ar}(4 \mathrm{p})}$ ratio along the reactor : in presence of a titanium target ; in absence of titanium.

( $P=5$ mbars, $P_{\mathrm{I}}=780 \mathrm{~W}$,

$$
\left.\left.P_{\mathrm{I}} / N=10 \times 10^{-17} \mathrm{~W} / \mathrm{mol} \text {., } D=0.67 \mathrm{l} / \mathrm{min}\right) .\right]
$$

température de la paroi, il constitue donc un autre paramètre clé de la chimie à l'interface, cependant le calcul du temps de séjour n'a pour l'instant pas été effectué.

Bien que la présence de la surface de 'titane augmente le temps de séjour global des espèces dans le réacteur, l'augmentation de la réactivité chimique ne peut être attribuée à ce seul facteur. En effet, la comparaison des courbes 6 et 8 montre qu'il faudrait que la présence du titane dans l'écoulement aug- mente d'environ $10 \mathrm{~ms}$ le temps de séjour (à $\tau_{\mathrm{s}}=30 \mathrm{~ms}$ ) pour que l'on puisse retrouver les valeurs du taux de conversion obtenues en présence de titane, ce qui est peu probable. Par ailleurs, des expériences effectuées dans le réacteur de plus grand diamètre, en présence de substrats de nature différente ont montré que le pouvoir catalytique dépend de la nature du métal et que le silicium inhibe la réaction de décomposition de $\mathrm{NH}_{3}$, les dimensions des substrats étant toutes égales (Réf. [16]).

La seule observation de la température de vibration ne permet pas de déterminer les mécanismes d'interaction plasma/surface et une analyse des intensités d'émissions de $\mathrm{N}_{2}\left(\mathrm{C}^{3} \Pi_{\mathrm{u}}\right)$ et de $\mathrm{NH}\left(\mathrm{A}^{3} \Pi\right)$ permet de préciser le rôle respectif des réactions en phase gazeuse et des réactions de surface.

La production de $\mathrm{NH}\left(\mathrm{A}^{3} \Pi\right)$ dans le réacteur peut s'effectuer selon plusieurs processus, dont certains font intervenir la production de $\mathrm{NH}_{2}$. Les coefficients d'Einstein pour les transitions $\mathrm{NH}_{2}\left(\tilde{\mathrm{A}}^{2} \mathrm{~A}_{1}\left(\begin{array}{lll}0 & 0 & 0\end{array}\right) \rightarrow\right.$ $\left.\tilde{\mathrm{X}}^{2} \mathrm{~B}_{1}(090)\right), \Sigma^{\mathrm{P}} \mathrm{Q}_{1, \mathrm{~N}}$ et $\mathrm{NH}\left(\mathrm{A}^{3} \Pi \rightarrow \mathrm{X}^{3} \Sigma^{-}\right)$respectivement de $1,19 \times 10^{4} \mathrm{~s}^{-1}$ et de $2,38 \times 10^{6} \mathrm{~s}^{-1}$ ont été calculés à partir des forces d'oscillateur (respectivement de 0,00807 (Réf. $[19,20]$ ) et $6,35 \times 10^{-5}$ (Réf. [24]). Le rapport des coefficients d'Einstein est de $5 \times 10^{-3}$, montrant ainsi que l'espèce $\mathrm{NH}_{2}^{*}$ est beaucoup plus difficile à détecter que $\mathrm{NH}^{*}$. Ainsi, le fait que nous n'ayons pas détecté $\mathrm{NH}_{2}^{*}$ dans nos conditions de décharge ne nous permet pas de conclure de façon définitive sur la formation de $\mathrm{NH}_{2}$. Cependant, d'Agostino et al. (Réf. [17]), dans des conditions similaires aux nôtres, bien que plus énergétiques (puissance variant de 1 à $4 \mathrm{~kW}$ ), ont

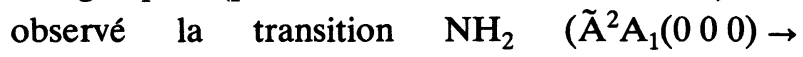
$\tilde{\mathrm{X}}^{2} \mathrm{~B}_{1}\left(\begin{array}{ll}0 & 12\end{array}\right)$ ) et ont pu montré que l'espèce $\mathrm{NH}_{2}$ est formé en début de réacteur et disparaît très rapidement. Par conséquent, nous pouvons admettre que, même si l'espèce $\mathrm{NH}_{2}$ est produite dans notre réacteur, elle disparaît très rapidement et ne constitue pas l'espèce intermédiaire majoritaire (contrairement à NH), notamment lors de l'interaction plasma/surface. Une étude plus approfondie est, cependant, prévue sur la production de cette espèce, durant laquelle nous utiliserons un amplificateur de signal en sortie du photomultiplicateur.

Outre ces processus, $\mathrm{NH}\left(\mathrm{A}^{3} \Pi\right)$ peut également être produit directement à partir de $\mathrm{NH}_{3}$ selon les mécanismes suivants :

$$
\begin{aligned}
&- \mathrm{NH}_{3}+\mathrm{e}^{-} \rightarrow \mathrm{NH}\left(\mathrm{A}^{3} \Pi\right)+2 \mathrm{H}\left({ }^{2} \mathrm{~S}\right)+\mathrm{e}^{-} \\
&- \mathrm{NH}_{3}+\mathrm{e}^{-} \rightarrow \mathrm{NH}\left(\mathrm{A}^{3} \Pi\right)+\mathrm{H}_{2}+\mathrm{e}^{-} \\
&- \mathrm{NH}_{3}+\mathrm{e}^{-} \rightarrow \mathrm{NH}\left(\mathrm{X}^{3} \Sigma^{-}\right)+\mathrm{H}_{2}+\mathrm{e}^{-} \\
& \text {puis }: \mathrm{NH}\left(\mathrm{X}^{3} \Sigma^{-}\right)+\mathrm{e}^{-} \rightarrow \mathrm{NH}\left(\mathrm{A}^{3} \Pi\right)+\mathrm{e}^{-}
\end{aligned}
$$$$
\text { (1) seuil d'énergie : } 12,2 \mathrm{eV}
$$

$7,8 \mathrm{eV}$

$3,8 \mathrm{eV}$

$3,8 \mathrm{eV}$ 
L'utilisation de l'argon en actinomètre pour cette espèce n'est théoriquement valable, compte tenu du seuil de réaction et de la forme de la section efficace, que si la réaction (1) est prépondérante. Cependant, en première approximation, puisque nous n'avons pas accès à d'autres diagnostics tels que la fluorescence induite par laser ou l'absorption laser, nous admettrons que les électrons d'énergie moyenne de $8 \mathrm{eV}$ et de $4 \mathrm{eV}$ suivent les mêmes variations en fonction des paramètres plasma que les électrons de $12-13 \mathrm{eV}$.

A partir de cette hypothèse, l'observation des variations de l'intensité d'émission de $\mathrm{NH}\left(\mathrm{A}^{3} \Pi\right)$ le long du réacteur (tenant compte des réactions de production et de disparition des radicaux $\mathrm{NH}\left(\mathrm{NH}(\mathrm{J})+\mathrm{NH}(\mathrm{J}) \rightarrow \mathrm{N}_{2}(v)+\mathrm{H}_{2}\right)$ ) nous permet de suggérer une interprétation de nos résultats.

La variation de l'intensité d'émission de $\mathrm{NH}\left(\mathrm{A}^{3} \Pi\right)$ le long du réacteur fait apparaître deux types de profils différents : l'un pour lequel l'intensité d'émission est maximale dans la zone centrale $\left(I_{\mathrm{NH}}\right.$ plasma $>I_{\mathrm{NH}}$ couche limite) (Fig. 11), et l'autre pour lequel l'intensité d'émission est maximale dans la couche limite $\left(I_{\mathrm{NH}}\right.$ plasma $<I_{\mathrm{NH}}$ couche lim.). C'est ainsi que pour un temps de séjour d'environ $20 \mathrm{~ms}, I_{\mathrm{NH}}$ couche limite $/ I_{\mathrm{NH}}$ plasma diminue de 1,5 à 0,5 lorsque $P_{\mathrm{I}} / N$ passe de 4 à $24 \times$ $10^{-17} \mathrm{~W} /$ molécule ; simultanément, la température de vibration augmente de 3000 à $3700 \mathrm{~K}$ dans la

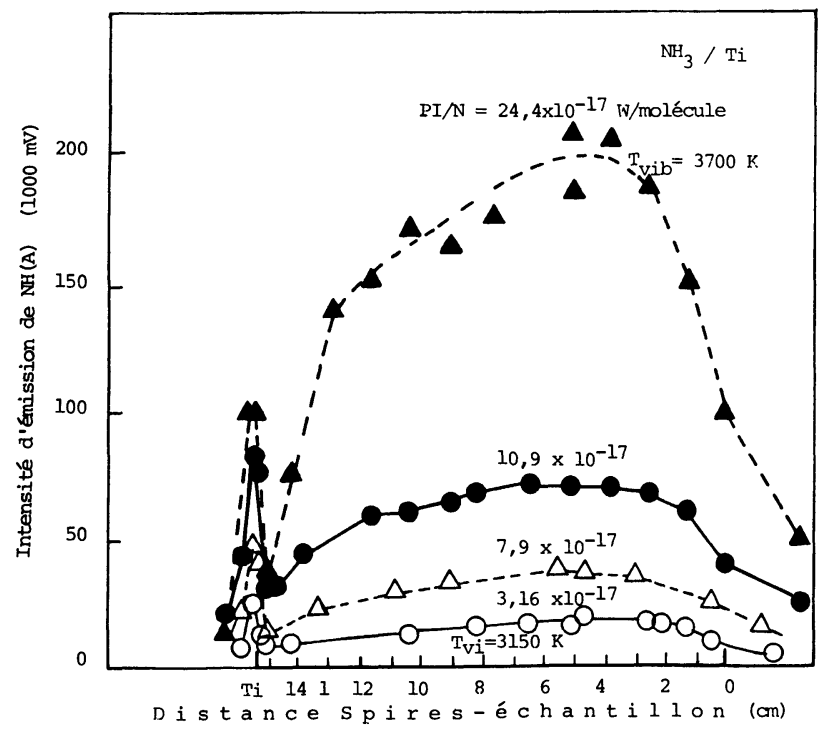

Fig. 11. - Influence de la densité d'énergie du plasma $\left(P_{\mathrm{I}} / N\right)$ sur le profil d'intensité d'émission du radical NH $\left(\mathrm{A}^{3} \Pi \rightarrow \mathrm{X}^{3} \Sigma^{-}\right.$) le long du réacteur. $P_{\mathrm{I}} / N$ (en $\mathrm{W} / \mathrm{molé}-$ cule) est égal à :

$24,4 \times 10^{-17} ; 10,9 \times 10^{-17} ; 7,9 \times 10^{-17} ; 3,16 \times 10^{-17}$.

[Role of the plasma energetic density $\left(P_{\mathrm{I}} / N\right)$ on the emission intensity profile of the $\mathrm{NH}$ radical ( ${ }^{3} \Pi \rightarrow \mathrm{X}^{3} \Sigma^{-}$), along the reactor. $P_{\mathrm{I}} / N$ (in W/molecule) is equate to :

$24.4 \times 10^{-17} ; 10.9 \times 10^{-17} ; 7.9 \times 10^{-17} ; 3.16 \times 10^{-17}$.] zone centrale. Si on maintient constante l'excitation vibrationnelle de la zone centrale à $3500 \mathrm{~K}, I_{\mathrm{NH}}$ couche limite $/ I_{\mathrm{NH}}$ plasma augmente de 0,5 à 2,5 lorsque le temps de séjour diminue de 45 à $4 \mathrm{~ms}$ (Fig. 12). Ceci nous amène à distinguer deux domaines de fonctionnement du réacteur :

$-I_{\mathrm{NH}}$ couche limite $/ I_{\mathrm{NH}}$ plasma $<1$, la décomposition de $\mathrm{NH}_{3}$ s'effectue principalement en phase gazeuse ; elle nécessite une forte excitation vibrationnelle du plasma et un temps de séjour important. La première étape de l'effet catalytique est la recombinaison des radicaux et/ou des atomes.

- $I_{\mathrm{NH}}$ couche limite $/ I_{\mathrm{NH}}$ plasma $>1$ : la faible excitation vibrationnelle du plasma et/ou le faible temps de séjour ne permet qu'une faible dissociation en phase homogène. L'augmentation du taux de décomposition de $\mathrm{NH}_{3}$, observé en présence de titane, peut être attribuée à trois types de phénomènes de surface distincts, sur lesquels nous reviendrons un peu plus tard.

Par ailleurs, pour un temps de séjour de $15 \mathrm{~ms}$, une augmentation de $P_{\mathrm{I}} / N$ de 11,8 à $46 \times$ $10^{-17} \mathrm{~W} /$ molécule se traduit par une augmentation de $I_{\mathrm{N}_{2}} / I_{\text {Ar }}$ d'un facteur 3,65 dans la zone centrale et d'un facteur 7,1 dans la couche limite (Figs. 13 et 14). La température de vibration augmente simultanément de 3300 à $4000 \mathrm{~K}$ dans la zone centrale et de 3800 à $4340 \mathrm{~K}$ dans la couche limite. L'augmen-

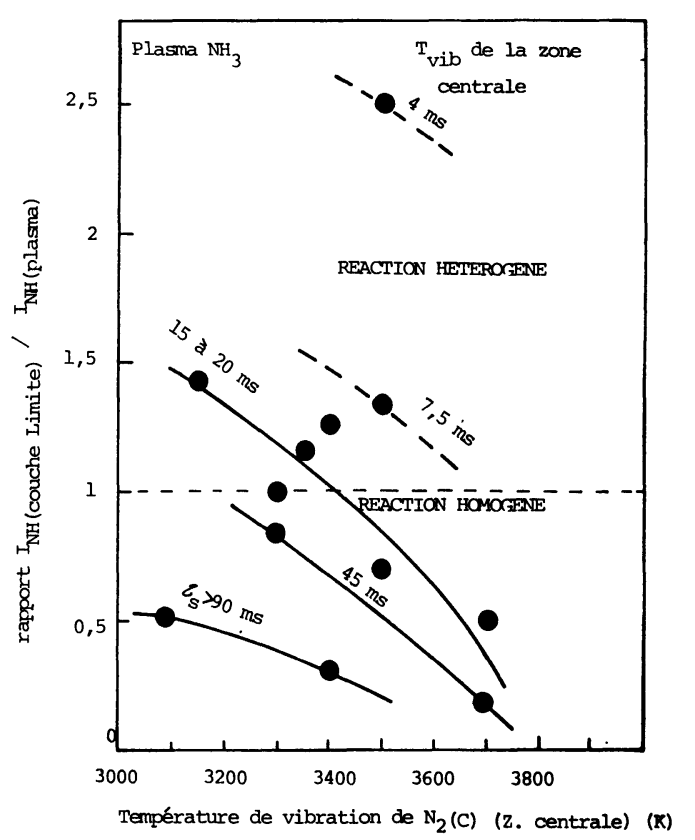

Fig. 12. - Influence de la température de vibration de la zone centrale du réacteur sur le rapport $I_{\mathrm{NH}}$ Couche Limite/ $I_{\mathrm{NH}}$ Plasma, à temps de séjour constant.

[Influence of the vibrational temperature of the plasma bulk on the $I_{\mathrm{NH}}$ Boundary layer $/ I_{\mathrm{NH}}$ Plasma ratio, for constant residence time.] 


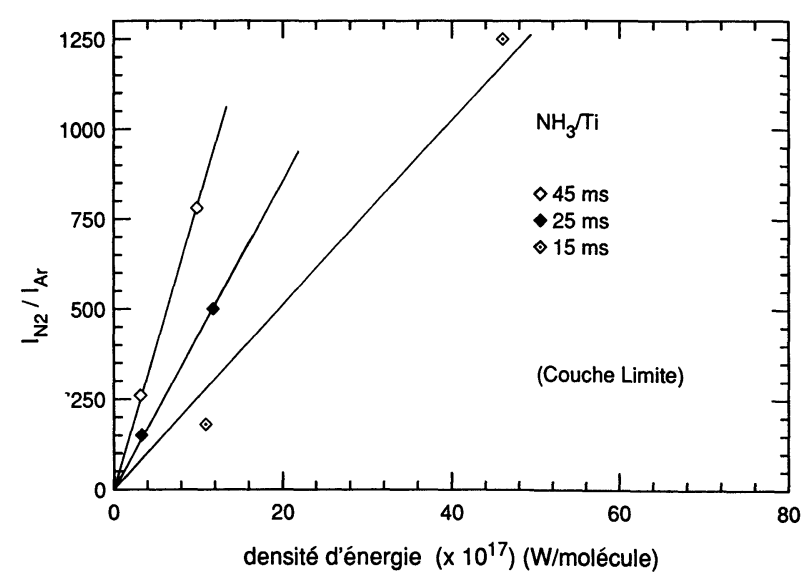

Fig. 13. - Influence de la densité d'énergie du plasma $\left(P_{\mathrm{I}} / N\right)$ sur le rapport $I_{\mathrm{N}_{2}} / I_{\mathrm{Ar}}$ dans la couche limite, à temps de séjour constant.

[Influence of the plasma energy density $\left(P_{\mathrm{I}} / N\right)$ on the $I_{\mathrm{N}_{2}} / I_{\mathrm{Ar}}$ ratio, in the boundary layer, for constant residence time.]

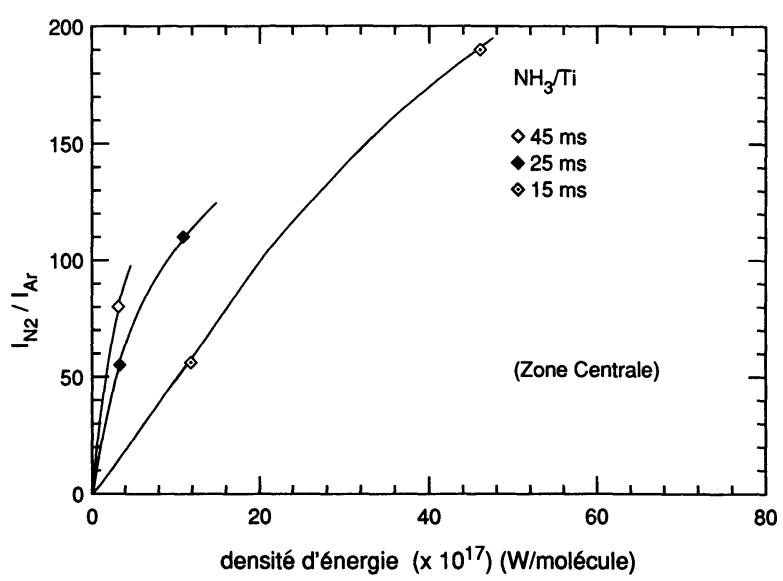

Fig. 14. - Influence de la densité d'énergie du plasma $\left(P_{\mathrm{I}} / N\right)$ sur le rapport $I_{\mathrm{N}_{2}} / I_{\mathrm{Ar}}$ dans la zone centrale, à temps de séjour constant.

[Influence of the plasma energy density $\left(P_{\mathrm{I}} / N\right)$ on the $I_{\mathrm{N}_{2}} / I_{\mathrm{Ar}}$ ratio, in the plasma bulk, for constant residence time.]

tation plus importante de $I_{\mathrm{N}_{2}} / I_{\mathrm{Ar}}$ dans la couche limite par rapport à la zone centrale confirme que la production de $\mathrm{N}_{2}(X, v)$ est prépondérante dans la couche limite suite aux processus de recombinaison catalytique. Par ailleurs, la variation de la température de vibration est, ainsi, directement reliée à la production d'azote moléculaire via la recombinaison des radicaux $\mathrm{NH}$ (ou des atomes $\mathrm{N}$ ) aussi bien dans la zone centrale que dans la couche limite.

Ces résultats sont, en ce qui concerne la phase gazeuse en accord avec les lois de Polanyi (Réf. [1]) puisque les étapes de recombinaison de $\mathrm{NH}$ ou de $\mathrm{N}$ constituent des étapes exothermiques, conduisant donc à des produits de réaction vibrationnellement excités. En ce qui concerne la couche limite, les résultats sont en accord avec les calculs de Wolken (Réf. [12]) qui a montré que, lors d'une étape de recombinaison-désorption sur une surface métallique, les molécules formées quittent la surface excitées vibrationnellement.

\section{Discussion ; conclusion.}

L'étude de la décomposition de $\mathrm{NH}_{3}$ en phase gazeuse homogène nous a permis d'établir une corrélation entre le taux de décomposition et l'excitation vibrationnelle du milieu. Cette corrélation est caractéristique d'un réacteur tubulaire contrôlé par une étape endothermique. Une modélisation de ce type de réacteur en phase plasma pourrait dès lors être envisagée en utilisant non plus la température du gaz mais une température de vibration rendant compte de l'excitation vibrationnelle de l'espèce réactive.

L'effet catalytique d'un échantillon de titane sur la décomposition a également été démontré. Il est associé à l'apparition d'une couche limite à l'interface plasma/surface caractérisée par une forte excitation vibrationnelle qui a pu être reliée à une augmentation de la production d'azote moléculaire dans cette zone. La contribution de cette réaction en phase hétérogène à la décomposition de $\mathrm{NH}_{3}$ est importante, cependant les mécanismes impliqués dépendent des paramètres de fonctionnement du réacteur.

En effet, pour une excitation vibrationnelle élevée et un temps de séjour important, la décomposition primaire de $\mathrm{NH}_{3}$ a majoritairement lieu en phase gazeuse. Il en résulte que le flux d'espèces entrant en interaction avec la surface est principalement constitué de radicaux $\mathrm{NH}$ et d'atomes d'azote plutôt que de molécules $\mathrm{NH}_{3}$. Le processus catalytique consiste alors en une chimisorption de ces espèces sur la surface, suivie d'une étape de diffusion des atomes d'azote dans le matériau (formation de nitrure) ou bien d'une étape de recombinaison-désorption, conduisant à la formation de molécules d'azote dans la couche limite.

Dans le cas d'une faible excitation vibrationnelle ou d'un faible temps de séjour, le flux gazeux entrant en interaction avec la surface est principalement constitué de molécules $\mathrm{NH}_{3}$ non dissociées qui peuvent se chimisorber sur la surface de titane. Dans le cas où $\mathrm{NH}_{3}$ est chimisorbé dissociativement, les atomes adsorbés se recombinent pour produire de l'azote moléculaire qui désorbe. Ainsi, un nouveau chemin réactionnel de dissociation de $\mathrm{NH}_{3}$ apparait, il correspond au mécanisme de catalyse hétérogène bien connu dans les milieux à l'équilibre thermodynamique. Cependant, dans ce cas de figure, deux autres mécanismes peuvent rendre compte de l'augmentation de la réactivité chimique due à la présence de la surface de titane. En effet, les 
électrons secondaires émis du métal dans la couche limite peuvent par collision avec les molécules non dissociées $\mathrm{NH}_{3}(X, v)$ conduire à leur dissociation (Réf. [14]) ; de plus, l'augmentation du temps de séjour des espèces dans la couche limite, due à la présence de la surface, augmente la probabilité pour que les molécules non dissociées se dissocient dans cette zone. Cependant, selon les conditions expérimentales, un de ces mécanismes deviendra prédominant, dans la mesure où ils sont respectivement fortement dépendants de la température du substrat, de la puissance, du débit et de la pression.

Quel que soit le mécanisme de l'étape initiale, des atomes d'azote sont chimisorbés sur la surface. L'étape suivante consiste en la recombinaison-désorption conduisant à la formation de molécules d'azote qui désorbent. Selon le mécanisme impliqué (dépendant de la température du substrat et de sa nature) :

$$
\text { Rideal : } \quad \mathrm{N}_{\text {gaz }}(\text { ou } \mathrm{NH})+\mathrm{N}_{\text {ads. }} \rightarrow \mathrm{N}_{2}(v)_{\text {gaz }}
$$

ou Langmuir Hinshelwood :

$$
\mathrm{N}_{\text {ads. }}+\mathrm{N}_{\text {ads. }} \rightarrow \mathrm{N}_{2}(v)_{\text {gaz }}
$$

les molécules quittent la surface avec une énergie plus ou moins importante. En effet, d'une part l'énergie libérée lors de la recombinaison-désorption est égale à respectivement $\Delta H^{0}=-604,5 \mathrm{~kJ} / \mathrm{mole}$ et à $\Delta H^{0}=-268,5 \mathrm{~kJ} / \mathrm{mole}$, selon le mécanisme et d'autre part, un partage non équitable de cette énergie a lieu entre le substrat et la molécule qui dépend également du mécanisme impliqué (Réf. [13]). Dans le cas du mécanisme de Rideal (plus probablement le cas du système $\mathrm{Ti} / \mathrm{NH}_{3}$ ), les molécules d'azote quittent la surface de titane en emportant une partie importante de cette énergie. Cette énergie étant canalisée sous forme d'excitation vibrationnelle, il s'ensuit que la réactivité chimique de la couche limite est augmentée (Réf. [5]). Ce mécanisme rétroactif de catalyse hétérogène est spécifique aux milieux plasmas hors équilibre.

\section{Bibliographie}

[1] Polanyi J. C., Acc. Chem. Res. 5 (1972) 161.

[2] Bergougnan M. P., Gicouel A., Amouroux J., Rev. Phys. Appl. 18 (1983) 335-46.

[3] D’Agostino R., Colaprico V., Cramarossa F., Plasma Chem. Plasma Process. 1 (1981) 365-375.

[4] Ricard A., Ann. Ch. Fr. 8 (1983) 303-318.

[5] Gicouel A., Thèse d'Etat, Université Paris VI (1987).

[6] Back R. A., Salahub D. R., Can. J. Chem. 45 (1967) 851.

[7] Tamagne C., Thèse Docteur Ingénieur, Université Paris VI (1976).

[8] Ricard A., Henrion G., Michel H., Gantois M., ISPC-8 2 (1987) 1055.

[9] Molinari E., Pure Appl. Chem. 39 (1974) 343.

[10] De Benedictis S., Gicquel A., Cramarossa F., ISPC-8 1 (1987) 631.

[11] Cramarossa F., Colaprico V., D'Agostino R., FERRARO G., ISPC-3 2 (1977).

[12] Wolken G., J. Chem. Phys. 68 (1978) 4338-4342.

[13] Halpern B., Rosner D. E., J. Chem. Soc. Faraday Trans. 160 (1978) 1883.

[14] Khait Y. L., CaRni U., Avni R., ISPC-6 3 (1983) 729.
[15] LAIDANI N., thèse de Docteur Ingénieur, Université Paris VI (1988).

[16] Gicquel A., Cavvadias S., Amouroux J., J. Phys. D. Appl. Phys. 19 (1986) 2013-2042.

[17] D'Agostino R., Cramarossa F., de Benedictis S., Ferraro G., Plasma Chem. Plasma Proc. 1 (1981) 19-35.

[18] Coburn J. W., Chen M., J. Appl. Phys. 51 (1980) 3134.

[19] Fairchild P. W., Smith G. P., Crosley D. R., Jeffries G. B., Chem. Phys. Lett. 107 (1984) 181.

[20] Garland N. L., Crosley D. R., soumis à J. Chem. Phys. (1988).

[21] Kelly R., Winkler C. A., Can. Chem. 37 (1959) 62-78.

[22] Young R. A., J. Chem. Phys. 34 (1961) 1292-1301.

[23] SAncier K. M., Fredericks W. J., WisW H., J. Chem. Phys. 37 (1962) 854-867.

[24] Kohse-Hoinghaus K., Davidson D. F., Chang A. Y., HANSON R. K., soumis à J.Q.S.R.T. (1988). 